\title{
Hecke transformation for orthogonal bundles and stability of Picard bundles
}

\author{
IndRANIL BisWAS AND TOMÁs L. GÓMEZ
}

\begin{abstract}
We define Hecke transformation for orthogonal bundles over a compact Riemann surface. Using the cycles on a moduli space of orthogonal bundles given by Hecke transformations, we prove that the projectivized Picard bundle on the moduli space is stable.
\end{abstract}

\section{Introduction}

Given a holomorphic vector bundle $F$ over a compact Riemann surface $X$, and a subspace $S_{x} \subset F_{x}$ in the fiber over a point $x$, the Hecke transformation produces a new vector bundle $E$ on $X[10,16]$. The vector bundle $E$ is the kernel of the natural quotient map $F \longrightarrow F_{x} / S_{x}$. Hecke transformation is a very useful tool to study the moduli space. For instance, they are used in computation of cohomologies of coherent sheaves on a moduli space of vector bundles [10]. They are also used in proving stability of various naturally associated bundles on a moduli space [3].

When $S_{x}$ varies among all subspaces of $F_{x}$ (the fiber of $F$ at $x$ ), with $x$ fixed, we get a family of vector bundles. Under suitable conditions for $F$, these Hecke transforms are stable vector bundles, so we obtain a morphism from the Grassmannian associated to $F_{x}$ to the moduli space of vector bundles. The image of this morphism is called a Hecke cycle.

An orthogonal bundle is a vector bundle $F$ together with a homomorphism $\psi: F \otimes F \longrightarrow M$, where $M$ is a line bundle, such that $\psi$ is symmetric and non-degenerate at every fiber. Equivalently, an orthogonal bundle can be thought of as a principal $\mathrm{GO}(r, \mathbb{C})$-bundle. Our aim here is systematically to construct Hecke transformations of orthogonal bundles. If $F$ is an orthogonal bundle over $X$ of rank $2 n$, and $S_{x} \subset F_{x}$ is an isotropic subspace of dimension $n$, then the vector bundle $E \longrightarrow X$ defined by the kernel of the homomorphism $F \longrightarrow F_{x} / S_{x}$ has an induced orthogonal structure. If the orthogonal form on $F$ takes values in a line bundle $M$, then the orthogonal form on $E$ takes values in $M \otimes \mathcal{O}_{X}(-x)$. Summing up, we start with a principal $\mathrm{GO}(2 n, \mathbb{C})$-bundle $F$ and a Lagrangian subspace of $F_{x}$, and we 
obtain another $\mathrm{GO}(2 n, \mathbb{C})$-bundle. If we let $S_{x}$ vary, we will obtain a family of $\mathrm{GO}(2 n, \mathbb{C})$-bundles. Under suitable conditions on $(F, \psi)$, all these bundles are stable, hence we obtain a morphism to the moduli space of stable orthogonal bundles, whose image is called a Hecke cycle.

For odd ranks, we consider vector bundles $F \longrightarrow X$ of rank $2 n+1$ equipped with a symmetric bilinear form $\psi$ which is nondegenerate on $X \backslash\{x\}$, and the annihilator $l_{x}$ of $F_{x}$ is of dimension one. For any isotropic subspace $\widetilde{S}_{x} \subset F_{x}$ of dimension $n+1$ (or, equivalently, for any isotropic subspace $S_{x} \subset F_{x} / l_{x}$ of dimension $n$ ), we construct an orthogonal bundle. As in the even case, we can define a morphism to the moduli space whose image is called a Hecke cycle. Note that in the odd case, in order to obtain a principal bundle, we start with an object which is not a principal bundle (the bilinear form on the fiber over $x$ is degenerate). From this point of view, a Hecke transformation, rather than a transformation between principal bundles, is better understood as a technique for producing interesting cycles in the moduli space.

As an application, we prove that the projectivized Picard bundle (see Section 5 for the definition) on a moduli space of orthogonal bundles is stable (Theorem 5.1).

In [4] we have considered symplectic Hecke transformations. At the end of this article we comment on the differences between the symplectic and orthogonal case.

\section{Preliminaries}

We fix a nondegenerate symmetric bilinear form $B$ on $\mathbb{C}^{r}, r \geq 3$. The symmetric matrix representing $B$ will also be denoted by $B$. Define the general orthogonal group $\mathrm{GO}(r, \mathbb{C})$ to be the group of all conformally orthogonal transformations, meaning

$$
\mathrm{GO}(r, \mathbb{C})=\left\{A \in \mathrm{GL}(r, \mathbb{C}): A^{t} B A=c B \text { for some } c \in \mathbb{C}^{*}\right\} .
$$

This group is an extension of $\mathbb{C}^{*}$ by the orthogonal group $\mathrm{O}(r, \mathbb{C})$

$$
1 \longrightarrow \mathrm{O}(r, \mathbb{C}) \longrightarrow \mathrm{GO}(r, \mathbb{C}) \stackrel{p}{\longrightarrow} \mathbb{C}^{*} \longrightarrow 1,
$$

where $p(A)$ is the constant $c$ in (2.1). It follows that

$$
\operatorname{det}(A)^{2}=p(A)^{r}
$$


Let $X$ be a compact connected Riemann surface. An orthogonal bundle on $X$ is a pair of the form $(E, \varphi)$, where

- $E \longrightarrow X$ is a holomorphic vector bundle, and

- $\varphi$ is a symmetric and nondegenerate holomorphic homomorphism

$$
\varphi: E \otimes E \longrightarrow L
$$

where $L \longrightarrow X$ is a holomorphic line bundle.

The homomorphism $\varphi$ induces an isomorphism $E \longrightarrow E^{\vee} \otimes L$, and this in turn produces an isomorphism

$$
\operatorname{det}(E)^{2} \stackrel{\sim}{\longrightarrow} L^{r}
$$

An isomorphism of orthogonal bundles

$$
(E, \varphi) \longrightarrow\left(E^{\prime}, \varphi^{\prime}\right)
$$

is a pair of holomorphic isomorphisms $\left(\alpha: E \stackrel{\sim}{\longrightarrow} E^{\prime}, \beta: L \stackrel{\sim}{\longrightarrow} L^{\prime}\right)$ such that the following diagram is commutative

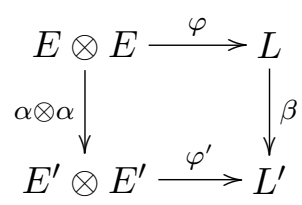

There is a canonical bijection between the isomorphism classes of principal $\mathrm{GO}(r, \mathbb{C})$-bundles and orthogonal bundles of rank $r$.

Let $(E, \varphi: E \otimes E \longrightarrow L)$ be an orthogonal bundle. If $F \subset E$ is a subsheaf, we define $F^{\perp}$ to be the kernel of the composition

$$
E \stackrel{\varphi}{\longrightarrow} E^{\vee} \otimes L \longrightarrow F^{\vee} \otimes L
$$

In other words, $F^{\vee}$ is the annihilator of $F$.

Lemma 2.1. Let $(E, \varphi: E \otimes E \longrightarrow L)$ be an orthogonal bundle on $X$, and let $F \subset E$ be a subbundle. 
(1) There is a short exact sequence on $X$

$$
0 \longrightarrow F^{\perp} \longrightarrow E \stackrel{\varphi}{\cong} E^{\vee} \otimes L \longrightarrow F^{\vee} \otimes L \longrightarrow 0
$$

hence $F^{\perp}$ is also a subbundle, so $\operatorname{rk} F^{\perp}=\operatorname{rk} E-\operatorname{rk} F$, and $\operatorname{deg} F^{\perp}=$ $\operatorname{deg} F+l\left(\frac{r}{2}-\operatorname{rk} F\right)$, where $l$ is the degree of $L$.

(2) There is a short exact sequence

$$
0 \longrightarrow F \cap F^{\perp} \longrightarrow F \oplus F^{\perp} \longrightarrow F+F^{\perp} \longrightarrow 0 .
$$

(3) We have an inclusion $F+F^{\perp} \subset\left(F \bigcap F^{\perp}\right)^{\perp}$, in particular, $\operatorname{rk}\left(F+F^{\perp}\right) \leq$ $\operatorname{rk}\left(F \cap F^{\perp}\right)^{\perp}$.

Proof. To prove (1), note that from (2.3) it follows that $\operatorname{deg} E=r l / 2$. Also, $F^{\vee}$ is a quotient bundle of $E^{\vee}$ because $F$ is a subbundle of $E$. Now (1) follows from the definition of $F^{\perp}$.

Part (2) is easy to check.

For (3), note that if $F_{1}$ is a subsheaf of $F_{2}$, then there is a natural inclusion $F_{2}^{\perp} \hookrightarrow F_{1}^{\perp}$. Since $F \bigcap F^{\perp} \subset F$ and $F \bigcap F^{\perp} \subset F^{\perp}$, we have $F^{\perp} \subset$ $\left(F^{\perp} \bigcap F\right)^{\perp}$, and $F=\left(F^{\perp}\right)^{\perp} \subset\left(F^{\perp} \bigcap F\right)^{\perp}$. Hence $F+F^{\perp} \subset\left(F^{\perp} \bigcap F\right)^{\perp}$.

A principal $\mathrm{GO}(r, \mathbb{C})$-bundle over a smooth complex projective curve $X$ is called stable (respectively, semistable) if for every reduction

$$
\sigma: X \longrightarrow P / Q
$$

to a (proper) maximal parabolic subgroup $Q \subset \mathrm{GO}(r, \mathbb{C})$,

$$
\operatorname{deg}\left(\sigma^{*} T_{\text {rel }}\right)>0 \quad\left(\text { respectively, } \operatorname{deg}\left(\sigma^{*} T_{\text {rel }}\right) \geq 0\right),
$$

where $T_{\text {rel }} \longrightarrow P / Q$ is the relative tangent bundle for the projection $P / Q \longrightarrow$ $X$ (see [13, page 129, Definition 1.1] and [13, page 131, Lemma 2.1]). In terms of orthogonal bundles, this condition is equivalent to the condition that for all isotropic subbundles $0 \neq E^{\prime} \subset E$,

$$
\frac{\operatorname{deg}\left(E^{\prime}\right)}{\operatorname{rk}\left(E^{\prime}\right)}<\frac{\operatorname{deg}(E)}{\operatorname{rk}(E)}\left(\operatorname{respectively}, \frac{\operatorname{deg}\left(E^{\prime}\right)}{\operatorname{rk}\left(E^{\prime}\right)} \leq \frac{\operatorname{deg}(E)}{\operatorname{rk}(E)}\right)
$$

we recall that $E^{\prime}$ is called an isotropic subbundle of $E$ if the restriction of $\varphi$ to $E^{\prime} \otimes E^{\prime} \subset E \otimes E$ is identically zero.

See $[14,15]$ for the construction of moduli spaces of semistable principal $\mathrm{GO}(r, \mathbb{C})$-bundles. We denote by $\mathcal{M}_{L}$ the moduli space of stable orthogonal bundles with values in the line bundle $L$. 
Lemma 2.2. If $(E, \varphi)$ is a semistable orthogonal bundle on $X$, then the underlying vector bundle $E$ is semistable.

Proof. The natural inclusion of $\operatorname{GO}(r, \mathbb{C})$ into $\mathrm{GL}(r, \mathbb{C})$ takes the center of $\mathrm{GO}(r, \mathbb{C})$ into the center of $\mathrm{GL}(r, \mathbb{C})$. Therefore, the lemma follows from [12, p. 285, Theorem 3.18].

\section{Hecke transformation for orthogonal bundles}

Let $X$ be a compact connected Riemann surface of genus $g=g(X) \geq 2$. Fix a point $x \in X$.

Proposition 3.1. Let $(F, \psi: F \otimes F \longrightarrow M)$ be an orthogonal bundle over $X$ of rank $r=2 n$. Let $S_{x} \subset F_{x}$ be an isotropic subspace of dimension $n$. Set $Q_{x}=F_{x} / S_{x}$, and let

$$
0 \longrightarrow E \longrightarrow F \longrightarrow Q_{x} \longrightarrow 0
$$

be the short exact sequence, where $F \longrightarrow F_{x} \longrightarrow Q_{x}$ is the natural projection. Then $E$ inherits a natural orthogonal structure $\varphi: E \otimes E \longrightarrow L$, where $L=$ $M(-x):=M \otimes_{\mathcal{O}_{X}} \mathcal{O}_{X}(-x)$.

Proof. Choose a local étale trivialization of $(F, \psi)$ around $x \in X$ such that $\psi$ is of the form

$$
\psi=\left(\begin{array}{lll}
0 & & 1 \\
& . & \\
1 & & 0
\end{array}\right)
$$

(so $\psi_{i, j}=0$ if $i+j \neq 2 n$, and $\psi_{i, 2 n-i}=1$ ) and $S_{x}$ is defined by the first $n$ vectors in the basis. The homomorphism $E \longrightarrow F$ is then locally defined by the matrix

$$
\left(\begin{array}{ll}
\mathbb{1}_{n} & \\
& t \mathbb{1}_{n}
\end{array}\right)
$$

(i.e., the diagonal matrix with the first $n$ entries equal to 1 and the last $n$ entries equal to $t$ ), where $t$ is a local parameter at $x \in X$. Therefore, the composition $E \otimes E \longrightarrow F \otimes F \longrightarrow M$ is

$$
\left(\begin{array}{lll}
0 & & t \\
& . \cdot & \\
t & & 0
\end{array}\right)
$$


so it vanishes at $x \in X$. Therefore, the homomorphism $E \otimes E \longrightarrow M$ factors through $L=M(-x)$, and then $\varphi: E \otimes E \longrightarrow L$ is of the form

$$
\left(\begin{array}{lll}
0 & & 1 \\
& . \cdot & \\
1 & & 0
\end{array}\right) .
$$

This completes the proof.

Let $(E, \varphi: E \otimes E \longrightarrow L)$ be an orthogonal bundle over $X$. Let

$$
\widehat{\varphi}: E \longrightarrow E^{\vee} \otimes L
$$

be the isomorphism given by $\varphi$. Define

$$
\varphi^{-1}:=\left(\widehat{\varphi}^{*}\right)^{-1}
$$

which produces a homomorphism $\varphi^{-1}: E^{\vee} \otimes E^{\vee} \longrightarrow L^{\vee}$. Note that $\left(E^{\vee}\right.$, $\left.\varphi^{-1}: E^{\vee} \otimes E^{\vee} \longrightarrow L^{\vee}\right)$ is an orthogonal bundle.

Proposition 3.2. Let $(E, \varphi: E \otimes E \longrightarrow L)$ be an orthogonal bundle over $X$ of rank $r=2 n$. Let $W$ be an isotropic subspace of dimension $n$ of $E_{x}^{\vee}$. Let $F^{\vee}$ be defined by the following short exact sequence

$$
0 \longrightarrow F^{\vee} \longrightarrow E^{\vee} \longrightarrow E_{x}^{\vee} / W \longrightarrow 0
$$

Then the orthogonal form $\varphi^{-1}$ on $E^{\vee}$ (see (3.2)) restricts to an orthogonal form

$$
\varphi^{-1}: F^{\vee} \otimes F^{\vee} \longrightarrow L^{\vee}(-x)
$$

on $F^{\vee}$.

Proof. The proposition follows by applying Proposition 3.1 to the orthogonal bundle $\left(E^{\vee}, \varphi^{*}: E^{\vee} \otimes E^{\vee} \longrightarrow L^{\vee}\right)$ and the subspace $W \subset E_{x}^{\vee}$.

Using (3.2), the orthogonal structure on $F^{\vee}$ in Proposition 3.2 produces an orthogonal structure

$$
F \otimes F \longrightarrow L(x)
$$

on $F$. 
Proposition 3.3. Let $F \longrightarrow X$ be a vector bundle of rank $r=2 n+1$ equipped with a symmetric bilinear form

$$
\psi: F \otimes F \longrightarrow M
$$

which induces a short exact sequence

$$
0 \longrightarrow F \longrightarrow F^{\vee} \otimes M \longrightarrow \mathbb{C}_{x} \longrightarrow 0
$$

where $\mathbb{C}_{x}$ is the skyscraper sheaf of length one supported over the point $x$. In other words, $\psi$ is nondegenerate everywhere except at $x$, and in an étale neighborhood of $x$, it is of the form

$$
\left(\begin{array}{lll}
0 & & \mathbb{1}_{n} \\
& t & \\
\mathbb{1}_{n} & & 0
\end{array}\right)
$$

(i.e., the $(n+1, n+1)$ th entry is $t$ and any other $(i, j)$ th entry $\left.i s \delta_{|i-j|, n+1}\right)$, where $t$ is a local parameter at $x \in X$. Let

$$
0 \longrightarrow l_{x} \longrightarrow F_{x} \longrightarrow F_{x}^{\vee} \otimes M_{x} \longrightarrow \mathbb{C}_{x} \longrightarrow 0
$$

be the exact sequence obtained by restricting the above short exact sequence to the point $x$. Let $S_{x}$ be an isotropic subspace of dimension $n$ of $F_{x} / l_{x}$. Define $Q_{x}:=\left(F_{x} / l_{x}\right) / S_{x}$, and consider the short exact sequence

$$
0 \longrightarrow E \longrightarrow F \longrightarrow Q_{x} \longrightarrow 0 .
$$

Then $\psi$ induces an orthogonal structure on $E$

$$
\varphi: E \otimes E \longrightarrow L:=M(-x)
$$

Proof. Choose a local trivialization such that $\psi$ is of the form in (3.5), and furthermore, the homomorphism $\beta: E \longrightarrow F$ is of the form

$$
\left(\begin{array}{ccc}
\mathbb{1}_{n} & & 0 \\
& 1 & \\
0 & & t \mathbb{1}_{n}
\end{array}\right) .
$$

Then the composition $E \otimes E \longrightarrow F \otimes F \longrightarrow M$ is

$$
\left(\begin{array}{ccc}
0 & & t \mathbb{1}_{n} \\
& t & \\
t \mathbb{1}_{n} & & 0
\end{array}\right) .
$$


This homomorphism $E \otimes E \longrightarrow M$ vanishes at $x \in M$, hence it factors through $L:=M(-x)$, inducing a homomorphism $\varphi: E \otimes E \longrightarrow L$. This $\varphi$ is symmetric and nondegenerate.

Proposition 3.4. Let $(E, \varphi: E \otimes E \longrightarrow L)$ be an orthogonal bundle over $X$ with $\operatorname{rk}(E)=2 n+1$, and let $W_{x} \subset E_{x}^{\vee}$ be an isotropic subspace of dimension $n$. Define $F^{\vee}$ using the short exact sequence

$$
0 \longrightarrow F^{\vee} \longrightarrow E^{\vee} \longrightarrow E_{x}^{\vee} / W_{x} \longrightarrow 0 .
$$

So $F \subset E(x):=E \otimes_{\mathcal{O}_{X}} \mathcal{O}_{X}(x)$. Consider the composition

$$
F \otimes F \hookrightarrow E(x) \otimes E(x) \stackrel{\varphi}{\longrightarrow} L(2 x)
$$

Its image lies in $M:=L(x) \subset L(2 x)$, and the corresponding symmetric bilinear form

$$
\psi: F \otimes F \longrightarrow M
$$

is everywhere nondegenerate except at the point $x$, where it is locally of the form

$$
\left(\begin{array}{lll}
0 & & \mathbb{1}_{n} \\
& t & \\
\mathbb{1}_{n} & & 0
\end{array}\right)
$$

Proof. With respect to a local trivialization of $E$ compatible with the filtration $W_{x} \subset S_{x} \subset E_{x}^{\vee}$, we have

$$
\varphi^{-1}=\left(\begin{array}{ccc}
0 & & \mathbb{1}_{n} \\
& 1 & \\
\mathbb{1}_{n} & & 0
\end{array}\right) \quad \text { and } f=\left(\begin{array}{ccc}
\mathbb{1}_{n} & & 0 \\
& 1 & \\
0 & & t \mathbb{1}_{n}
\end{array}\right)
$$

where $f$ is the homomorphism $F^{\vee} \hookrightarrow E^{\vee}$. Therefore,

$$
\psi^{\prime}=\left(\begin{array}{ccc}
0 & & t \mathbb{1}_{n} \\
& 1 & \\
t \mathbb{1}_{n} & & 0
\end{array}\right) \quad \text { and }\left(\psi^{\prime}\right)^{-1}=\frac{1}{t}\left(\begin{array}{ccc}
0 & & \mathbb{1}_{n} \\
& t & \\
\mathbb{1}_{n} & & 0
\end{array}\right)
$$

Since $\left(\psi^{\prime}\right)^{-1}$ has a pole of order one at $x \in X$, it induces a homomorphism with values on $M:=L(x)$; this induced homomorphism has the required properties.

We will also need to consider vector bundles $F$ with a symmetric bilinear tensor $\psi: F \otimes F \longrightarrow M$ which can be degenerate at some point (as in 
Proposition 3.3). In this case we still say that a subsheaf $F^{\prime} \subset F$ is isotropic if the restriction of $\psi$ to $F^{\prime} \otimes F^{\prime}$ is identically zero.

Following [11], we define:

Definition 3.1. Let $k, l$ be integers. A symmetric bilinear tensor $(E, \varphi)$ is $(k, l)$-stable (respectively, $(k, l)$-semistable) if for all isotropic subbundles $E^{\prime}$ of it of positive rank, the following inequality holds:

$$
\frac{\operatorname{deg}\left(E^{\prime}\right)+k}{\operatorname{rk}\left(E^{\prime}\right)}<\frac{\operatorname{deg}(E)+k-l}{\operatorname{rk}(E)}
$$

(respectively, $\left.\frac{\operatorname{deg}\left(E^{\prime}\right)+k}{\operatorname{rk}\left(E^{\prime}\right)} \leq \frac{\operatorname{deg}(E)+k-l}{\operatorname{rk}(E)}\right)$.

If $k=l=0$ and $(E, \varphi)$ is an orthogonal bundle (meaning $\varphi$ is nondegenerate), then the above definition coincides with the definition of (semi)stable orthogonal bundles.

For any $t \in \mathbb{R}$, let $[t]$ be the unique integer such that $t \leq[t]<t+1$.

Lemma 3.1. Let $(E, \varphi)$ be a $(n, n)$-stable orthogonal bundle of rank $r$, where $n=[r / 2]$. Let $(F, \psi)$ be obtained from $(E, \varphi)$ as in Proposition 3.2 or Proposition 3.4 (depending on the parity of $r$ ), and let $\left(E^{\prime}, \varphi^{\prime}\right)$ be obtained from $(F, \psi)$ as in Proposition 3.1 or Proposition 3.3. Then $\left(E^{\prime}, \varphi^{\prime}\right)$ is a stable orthogonal bundle.

Proof. By construction, we have a diagram

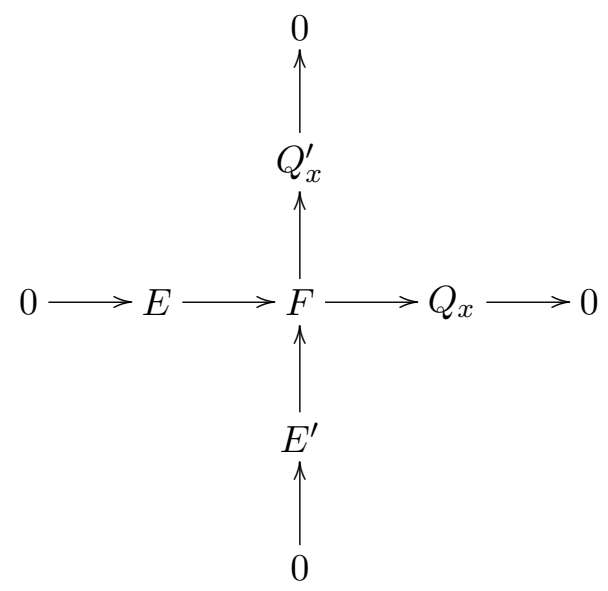


Let $H \subset E^{\prime}$ be an isotropic subbundle. Then $H \bigcap E$ is an isotropic subsheaf of $E$, and $\operatorname{deg} H-n \leq \operatorname{deg} H \bigcap E$, because the length of $Q_{x}$ is $n$. Therefore,

$$
\frac{\operatorname{deg} H}{\operatorname{rk} H} \leq \frac{\operatorname{deg} H \cap E+n}{\operatorname{rk} H} \leq \frac{\operatorname{deg} E}{\operatorname{rk} E}=\frac{\operatorname{deg} E^{\prime}}{\operatorname{rk} E^{\prime}}
$$

where the second inequality follows from the $(n, n)$-stability condition on $E$.

Proposition 3.5. Let $\mathcal{M}_{L}$ be the moduli space of stable orthogonal bundles of rank $r$ and degree $d$ (the line bundle $L$ is fixed). Assume that $g(X)>$ $n$ where $n=[r / 2]$. Then the subset of $\mathcal{M}_{L}$ corresponding to $(n, n)$-stable bundles is nonempty Zariski open.

Proof. There is a finite set of pairs $\left(r^{\prime}, d^{\prime}\right) \in \mathbb{N} \times \mathbb{Z}$ such that there is a stable orthogonal bundle $(E, \varphi) \in \mathcal{M}_{L}$ which has a quotient of rank $r^{\prime}$ and degree $d^{\prime}$ contradicting the $(n, n)$-stability condition. This and the properness of the Quot scheme together imply that the condition of being $(n, n)$-stable is Zariski open.

The dimension of $\mathcal{M}_{L}$ is calculated using [15, Theorem 5.9] and subtracting $g(X)$, because the line bundle $L$ where the orthogonal form takes values is fixed. More precisely,

$$
\begin{aligned}
\operatorname{dim} \mathcal{M}_{L} & =(g(X)-1) \operatorname{dim} \mathrm{GO}(r, \mathbb{C})+\operatorname{dim} Z(\mathrm{GO}(r, \mathbb{C}))-g(X) \\
& =(g(X)-1) \frac{r^{2}-r}{2}
\end{aligned}
$$

We will now estimate the dimension of the subset of the moduli space $\mathcal{M}_{L}$ corresponding to orthogonal bundles which are not $(n, n)$-stable.

Let $(E, \varphi)$ be such an orthogonal bundle, and let $P$ be the corresponding principal $\mathrm{GO}(r, \mathbb{C})$-bundle. An isotropic subbundle $H \subset E$ gives a reduction of structure group $P^{Q} \subset P$ to a maximal parabolic subgroup $Q \subset \operatorname{GO}(r, \mathbb{C})$.

Given a principal $Q$-bundle $E_{Q} \longrightarrow X$, we get a principal $\operatorname{GO}(r, \mathbb{C})$ bundle $E_{Q} \times^{Q} \operatorname{GO}(r, \mathbb{C})$ by extending the structure group. Since the stability condition is open, a deformation of $P^{Q}$ as a principal $Q$-bundle will give a deformation of $P$ which is stable but not $(n, n)$-stable. Furthermore, any deformation of $P$ which is not $(n, n)$-stable must be of this form for some parabolic subgroup $Q \subset \mathrm{GO}(r, \mathbb{C})$. 
The tangent space of these deformations has dimension $h^{1}\left(P^{Q}(\mathfrak{q})\right)-$ $g(X)$, where $\mathfrak{q}$ is the Lie algebra of $Q$, and $P^{Q}(\mathfrak{q})$ is the adjoint vector bundle of $P^{Q}$. We subtract $g(X)$ because the line bundle $L$, where the orthogonal form takes values, is fixed.

We claim that $h^{0}\left(P^{Q}(\mathfrak{q})\right)=1$. Indeed, on one hand we have

$$
h^{0}\left(P^{Q}(\mathfrak{q})\right) \geq \operatorname{dim} \mathfrak{z}(\mathfrak{q})=1
$$

where $\mathfrak{z}(\mathfrak{q})$ is the center of the Lie algebra $\mathfrak{q}$. On the other hand, since $P$ is a stable principal $\mathrm{GO}(r, \mathbb{C})$-bundle, we have

$$
H^{0}(X, P(\mathfrak{g o}(r, \mathbb{C})))=\mathfrak{z}(\mathfrak{g o}(r, \mathbb{C}))
$$

where $P(\mathfrak{g} \mathfrak{o}(r, \mathbb{C}))$ is the adjoint bundle of $P$, and $\mathfrak{z}(\mathfrak{g} \mathfrak{o}(r, \mathbb{C})) \subset \mathfrak{g} \mathfrak{o}(r, \mathbb{C})$ is the center [13, page 136, Proposition 3.2]. Since $\mathfrak{q}$ is a submodule of the $Q$-module $\mathfrak{g} \mathfrak{o}(r, \mathbb{C})$, the vector bundle $P^{Q}(\mathfrak{q})$ is a subbundle of the adjoint vector bundle $P(\mathfrak{g o}(r, \mathbb{C}))$. Therefore,

$$
h^{0}\left(P^{Q}(\mathfrak{q})\right) \leq h^{0}(P(\mathfrak{g} \mathfrak{o}(r, \mathbb{C})))=\operatorname{dim} \mathfrak{z}(\mathfrak{g} \mathfrak{o}(r, \mathbb{C}))=1
$$

Combining this with $(3.8)$ it follows that $h^{0}\left(P^{Q}(\mathfrak{q})\right)=1$.

Using Riemann-Roch,

$$
h^{1}\left(P^{Q}(\mathfrak{q})\right)-g(X)=-\operatorname{deg}\left(P^{Q}(\mathfrak{q})\right)+\left(\operatorname{rk}\left(P^{Q}(\mathfrak{q})\right)-1\right) \cdot(g(X)-1) .
$$

Therefore, by Lemma 3.2, the codimension $\operatorname{codim} Z$ of the subscheme $Z \subset$ $\mathcal{M}_{L}$ defined by all orthogonal bundles which are not $(n, n)$-stable satisfies the inequality

$$
\begin{aligned}
\operatorname{codim} Z & \geq \operatorname{dim} \mathcal{M}_{L}-\left(h^{1}\left(P^{Q}(\mathfrak{q})\right)-g\right) \\
& =\operatorname{dim} \mathrm{O}(r)(g-1)+\operatorname{deg} P^{Q}(\mathfrak{q})-\left(\operatorname{rk} P^{Q}(\mathfrak{q})-1\right)(g-1) \\
& =\operatorname{deg} P^{Q}(\mathfrak{q})+(\operatorname{dim} \mathrm{O}(r)-\operatorname{dim} \mathfrak{q}+1)(g-1) \\
& =\left(\frac{e}{s}-\frac{d}{r}\right) s(r-s-1)-\frac{3 s^{2}-2 r s+s}{2}(g-1) \\
& \geq-n(r-s-1)-\frac{3 s^{2}-2 r s+s}{2}(g-1) .
\end{aligned}
$$


In the last line we have used the fact that $H \subset E$ contradicts the $(n, n)-$ stability condition, which translates into the inequality $e / s-d / r \geq-n / s$.

We have to show that the expression in the last line is positive. We first assume that $r$ is odd, so we substitute $r=2 n+1$. The first summand in the last line is

$$
f_{1}(s):=-n(2 n+1-s-1)=-2 n^{2}+n s \geq-2 n^{2}+n
$$

since $s \geq 1$. The second summand becomes

$$
f_{2}(s):=-\left(\frac{3 s^{2}}{2}-2 n s-\frac{s}{2}\right)(g-1)=-\frac{3}{2}\left(s-\left(\frac{4 n+1}{3}\right)\right) s(g-1) .
$$

The graph of the function $f_{2}(s)$ is a parabola, which is zero for $s=0$ and $s=$ $(4 n+1) / 3$, and has a maximum for $s=(4 n+1) / 6$. Therefore, the minimum value in the interval $1 \leq s \leq n$ is attained at $s=1$. Consequently,

$$
f_{2}(s) \geq f_{2}(1)=-\frac{3}{2}\left(1-\left(\frac{4 n+1}{3}\right)\right)(g-1)
$$

Finally,

$$
\operatorname{codim} Z \geq f_{1}(s)+f_{2}(s) \geq-2 n^{2}+n-(1-2 n)(g-1),
$$

and this is positive when $g>n+1$.

We now assume that $r$ is even, so we set $r=2 n$. The first summand is then

$$
f_{1}(s):=-n(2 n-s-1) \geq-n(2 n-2)
$$

since $s \geq 1$, and the second summand becomes

$$
f_{2}(s):=-\left(\frac{3 s^{2}}{2}-2 n s+\frac{s}{2}\right)(g-1)=-\frac{3}{2}\left(s-\left(\frac{4 n-1}{3}\right)\right) s(g-1) .
$$

The graph of the function $f_{2}(s)$ is a parabola, which is zero for $s=0$ and $s=(4 n-1) / 3$, and it is positive in between these values. Note that $(4 n-1) / 3>n$, because $n>1$. (Recall that we are assuming $r>2$. Since 
we are in the even case $r=2 n$, this means that $n>1$.) Hence the minimum value in the interval $1 \leq s \leq n$ is attained at $s=1$. Therefore,

$$
f_{2}(s) \geq f_{2}(1)=-\frac{3}{2}\left(1-\left(\frac{4 n-1}{3}\right)\right)(g-1)=(2 n-2)(g-1) .
$$

Finally,

$$
\operatorname{codim} Z \geq f_{1}(s)+f_{2}(s) \geq(2 n-2)(g-1-n),
$$

and it is positive when $g>n+1$.

Lemma 3.2. Let $(E, \varphi: E \otimes E \longrightarrow L)$ be an orthogonal bundle with $\mathrm{rk} E=$ $r$ and $\operatorname{deg} E=d$. Let $P$ be the corresponding principal $\mathrm{GO}(r, \mathbb{C})$-bundle. Let $H \subset E$ be an isotropic subbundle with $\operatorname{deg} H=e$ and $\operatorname{rk} H=s$. Let $P^{Q} \subset P$ be the corresponding reduction of structure group to a maximal parabolic subgroup $Q \subset \mathrm{GO}(r, \mathbb{C})$. Then

$$
\begin{aligned}
\operatorname{deg} P^{Q}(\mathfrak{q}) & =\left(\frac{e}{s}-\frac{d}{r}\right) s(r-s-1) \\
\operatorname{dim} \mathfrak{q} & =\frac{r^{2}-r}{2}-\frac{2 r s-3 s^{2}-s}{2}+1 .
\end{aligned}
$$

Proof. Let $P^{Q}(\mathfrak{g l}(r, \mathbb{C}))$ be the Lie algebra bundle associated to $P^{Q}$ and the adjoint action of $Q$ on $\mathfrak{g l}(r, \mathbb{C})$; so, $P^{Q}(\mathfrak{g l}(r, \mathbb{C})) \cong E^{\vee} \otimes E$. Since $\mathfrak{q}$ is a $\operatorname{GO}(r, \mathbb{C})$-submodule of $\mathfrak{g l}(r, \mathbb{C})$, the vector bundle $P^{Q}(\mathfrak{q})$ is a subbundle of $E^{\vee} \otimes E$. The subbundle $P^{Q}(\mathfrak{q})$ preserves the filtration

$$
H \subset H^{\perp} \subset E
$$

where $H^{\perp}$ is the orthogonal bundle to $H$ with respect to the orthogonal structure $\varphi$. Therefore, we have rk $H+\mathrm{rk} H^{\perp}=r$.

Let $L(Q)$ be the Levi quotient of the parabolic subgroup $Q$. Fixing

$$
T \subset B \subset Q
$$

where $T$ is a maximal torus of $\operatorname{GO}(r, \mathbb{C})$ and $B$ a Borel subgroup of $\operatorname{GO}(r, \mathbb{C})$, the quotient $L(Q)$ of $Q$ can be realized as a subgroup of $Q$. In fact, the maximal connected $T$-invariant reductive subgroup of $Q$ is identified with $L(Q)$. Fix such a subgroup of $Q$. This subgroup of $Q$ will also be denoted by $L(Q)$; it will be called the Levi subgroup.

Let $P^{L(Q)}$ denote the principal $L(Q)$-bundle obtained by extending the structure group of $P^{Q}$ using the projection of $Q$ to its Levi quotient $L(Q)$. 
Let $P^{L(Q)}(Q)$ be the principal $Q$-bundle obtained by extending the structure group of $P^{L(Q)}$ using the inclusion of the Levi subgroup $L(Q) \subset Q$ that has been fixed. The principal $Q$-bundle $P^{L(Q)}(Q)$ is topologically isomorphic to the principal $Q$-bundle $P^{Q}$. Hence the two adjoint bundles $P^{Q}(\mathfrak{q})$ and $P^{L(Q)}(Q)(\mathfrak{q})$ are topologically isomorphic. Therefore, to calculate the degree of $P^{Q}(\mathfrak{q})$, we can replace $P^{Q}$ by $P^{L(Q)}(Q)$. In other words, we can assume that $P^{Q}$ admits a reduction of structure group $P^{L(Q)} \subset P^{Q}$ to the Levi subgroup $L(Q) \subset Q$. Fix a reduction of structure group $P^{L(Q)} \subset P^{Q}$ to $L(Q)$.

The filtration (3.9) splits using the reduction of structure group $P^{L(Q)} \subset$ $P^{Q}$. In other words, we have an isomorphism,

$$
E \cong H \oplus\left(H^{\perp} / H\right) \oplus\left(E / H^{\perp}\right)
$$

Using (3.10), a locally defined section of the adjoint bundle $P^{Q}(\mathfrak{q})$ has the form

$$
A=\left(\begin{array}{lll}
\alpha & \beta & \gamma \\
0 & \delta & \epsilon \\
0 & 0 & \eta
\end{array}\right) .
$$

The isomorphism of vector bundles

$$
E \longrightarrow E^{\vee} \otimes L
$$

induced by the orthogonal structure $\varphi$ has the property that the composition

$$
H^{\perp} \hookrightarrow F \longrightarrow F^{\vee} \otimes M \longrightarrow H^{\vee} \otimes M
$$

vanishes. Consequently, we have an induced isomorphism

$$
F / H^{\perp} \cong H^{\vee} \otimes M
$$

which we will denote by $\mathbb{1}$. Also note that $\varphi$ induces an orthogonal structure on the vector bundle $H^{\perp} / H$. Let

$$
\varphi^{\prime}: H^{\perp} / H \longrightarrow\left(H^{\perp} / H\right)^{\vee} \otimes M
$$

be the isomorphism induced by this orthogonal structure. 
Now, using (3.10), the isomorphism in (3.12) has the form

$$
\left(\begin{array}{lll}
0 & 0 & \mathbb{1} \\
0 & \varphi^{\prime} & 0 \\
\mathbb{1} & 0 & 0
\end{array}\right)
$$

where $\varphi^{\prime}$ is defined above.

A parabolic subalgebra $\mathfrak{q}$ of $\mathfrak{g o}(r, \mathbb{C})$ is of the form $\mathfrak{q}=\mathfrak{q}^{\prime} \oplus \mathbb{C}$, where $\mathfrak{q}^{\prime}$ is a parabolic subalgebra of $\mathfrak{s o}(r, \mathbb{C})=\operatorname{Lie}(\mathrm{SO}(r, \mathbb{C}))$, and the summand $\mathbb{C}$ is the center of $\mathfrak{g o}(r, \mathbb{C})$. This decomposition is preserved by the adjoint action of $\mathrm{GO}(r, \mathbb{C})$. Therefore,

$$
P^{Q}(\mathfrak{q})=P^{Q}\left(\mathfrak{q}^{\prime}\right) \oplus \mathcal{O}_{X}
$$

The condition that the local section $A$, defined in (3.11), of $P(\mathfrak{g l}(2 n, \mathbb{C}))$ lies in $P^{Q}\left(\mathfrak{q}^{\prime}\right)$ is equivalent to the condition that

$$
\varphi \circ A=\left(\begin{array}{ccc}
0 & 0 & \eta \\
0 & \varphi \circ \delta & \varphi \circ \epsilon \\
\alpha & \beta & \gamma
\end{array}\right): E \longrightarrow E^{\vee} \otimes L
$$

is skew-symmetric, where $\varphi$ is defined in (3.13). Clearly, $\varphi \circ A$ is skewsymmetric if and only if the following three conditions hold:

(1) $\eta=-\alpha^{t}$,

(2) $\epsilon=-\varphi^{\prime-1} \circ \beta^{t}$, and

(3) the homomorphisms $\gamma$ and $\varphi^{\prime} \circ \delta$ are skew-symmetric.

Therefore, there is an isomorphism

$$
\begin{aligned}
P^{Q}\left(\mathfrak{q}^{\prime}\right) \cong & \operatorname{End}(H) \oplus\left(\left(\frac{H^{\perp}}{H}\right)^{\vee} \otimes H\right) \oplus\left(\left(\bigwedge^{2} H\right) \otimes M^{\vee}\right) \\
& \oplus\left(\bigwedge^{2}\left(\frac{H^{\perp}}{H}\right)^{\vee} \otimes M\right)
\end{aligned}
$$

defined by

$$
A \longmapsto\left(\alpha, \beta, \gamma, \varphi^{\prime} \circ \delta\right) .
$$

From this isomorphism it follows immediately that $\operatorname{rk}\left(P^{Q}(\mathfrak{q})\right)=\operatorname{dim} \mathfrak{q}$. 
Using (3.14) we further have

$$
\operatorname{deg}\left(P^{Q}(\mathfrak{q})\right)=\operatorname{deg}\left(P^{Q}\left(\mathfrak{q}^{\prime}\right)\right)=\left(\frac{e}{s}-\frac{d}{r}\right) s(r-s-1) .
$$

This completes the proof.

\section{Hecke cycles}

In this section, we will construct a family of orthogonal bundles using the constructions introduced in Proposition 3.1 and Proposition 3.3. If the rank is even, then the starting point will be an orthogonal bundle. If the rank is odd, then the starting point will be a vector bundle with a symmetric bilinear form singular over a fixed point $x$ (as in Proposition 3.3).

We will first describe the even case $r=2 n$.

Let $(F, \psi: F \otimes F \longrightarrow L(x))$ be a $(0, n)$-stable orthogonal bundle over $X$ of rank $r=2 n$ (as in Proposition 3.1). So $\psi$ takes values in the line bundle $L(x)$, where $x \in X$ is the fixed point. We assume that $L$ is such that

$$
\operatorname{deg} F>(2 g-2) r+r+n
$$

Take a quotient $F_{x} \longrightarrow Q_{x}$ over the given point $x \in X$ with $\operatorname{dim} Q_{x}=n$. We obtain the following commutative diagram of coherent sheaves on $X$ :

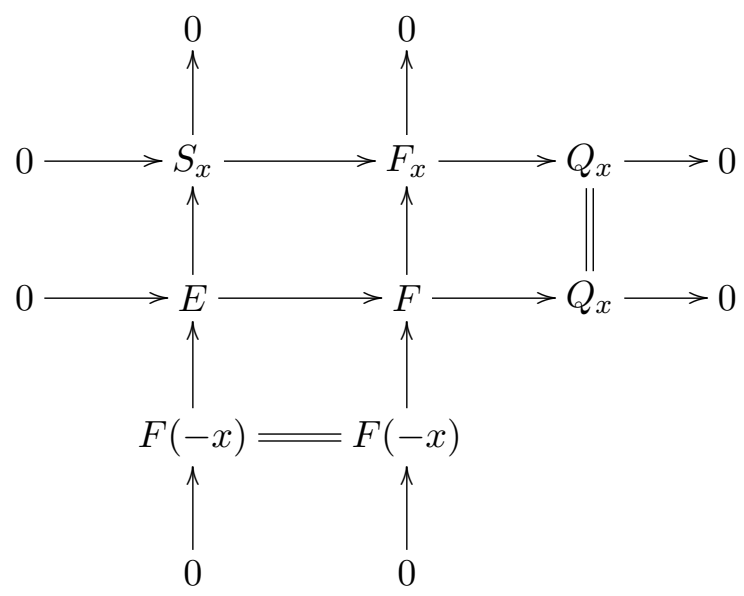

All the quotients of $F_{x}$ with the property that the corresponding kernel $S_{x}$ is an isotropic subspace of $F_{x}$ are parameterized by

$$
Y=\operatorname{Gr}_{\text {iso }, n}\left(F_{x}\right) \cong \operatorname{GO}(2 n, \mathbb{C}) / Q,
$$


where $Q \subset \operatorname{Gp}(2 n, \mathbb{C})$ is the parabolic subgroup preserving a fixed isotropic subspace of dimension $n$ of $\mathbb{C}^{2 n}$.

All these Hecke transformations parameterized by $Y$ combine to form the following commutative diagram of sheaves on $X \times Y$

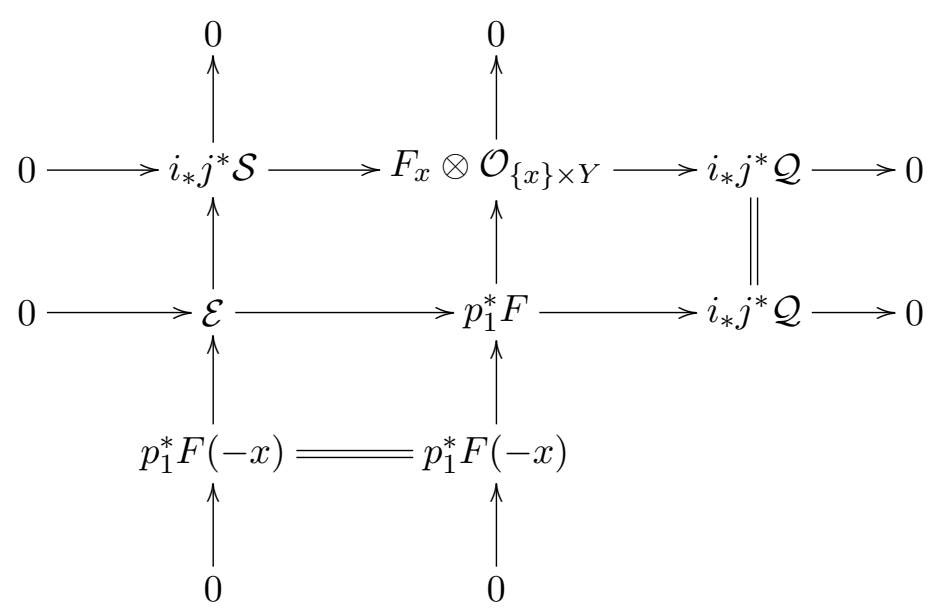

where $i:\{x\} \times Y \longrightarrow X \times Y$ and $j:\{x\} \times Y \longrightarrow Y$ are the natural inclusion and isomorphism, respectively; here $p_{1}$ is the natural projection of $X \times Y$ to $X$. The vector bundles $\mathcal{S}$ and $\mathcal{Q}$ are respectively the universal subbundle and quotient bundle of $F_{x}$ over $Y \cong \mathrm{GO}(2 n, \mathbb{C}) / Q$.

If the given orthogonal bundle $(F, \psi: F \otimes F \longrightarrow L(x))$ is $(0, n)$-stable, then all the orthogonal bundles constructed by Hecke transformations from $(F, \psi)$ are stable (see Lemma 3.1). The resulting classifying morphism

$$
\Psi: Y \longrightarrow \mathcal{M}_{L}
$$

will be called the Hecke morphism, where $\mathcal{M}_{L}$ as before is the moduli space of orthogonal bundles of rank $2 n$ with values in $L$.

We claim that $H^{1}(X, F(-x))=0$. Indeed, after twisting the middle row of (4.2) with $\mathcal{O}_{X}(-x)$, the associated long exact sequence gives a surjection

$$
H^{1}(E(-x)) \longrightarrow H^{1}(F(-x))
$$

Since $(F, \psi: F \otimes F \longrightarrow L(x))$ is $(0, n)$-stable, the orthogonal bundle $(E, \varphi$ : $E \otimes E \longrightarrow L)$ is stable, therefore the underlying vector bundle $E$ is 
semistable by Proposition 2.2. We have

$$
h^{1}(E(-x))=h^{0}\left(K_{X}(x) \otimes E^{\vee}\right)
$$

(Serre duality). On the other hand, $\operatorname{deg} E=\operatorname{deg} F-n>(2 g-2) r+r$ (see (4.1)). Therefore, $K_{X}(x) \otimes E^{\vee}$ is a semistable vector bundle of negative degree, so it cannot have nonzero sections. This proves the claim that $H^{1}$ $(X, F(-x))=0$.

Applying $p_{2 *}$ to (4.3), where $p_{2}$ is the natural projection of $X \times Y$ to $Y$, we obtain the following commutative diagram of sheaves on $Y$ :

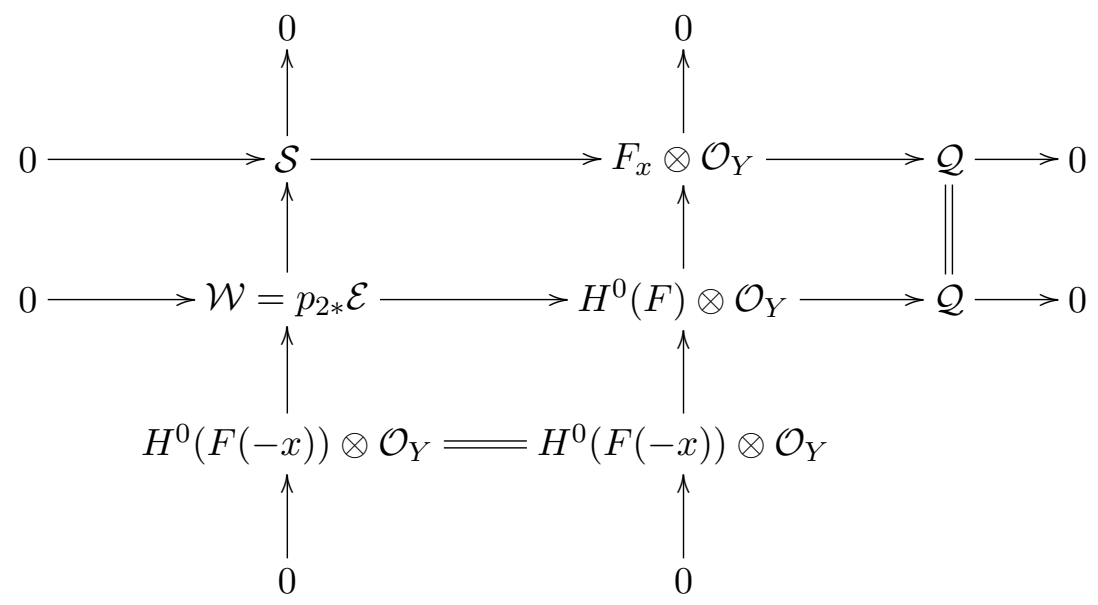

Now assume that $r=2 n+1$.

Let $F \longrightarrow X$ be a vector bundle of rank $r=2 n+1$ with a symmetric bilinear form

$$
\psi: F \otimes F \longrightarrow L(x)
$$

which induces a short exact sequence as in (3.4) (so $(F, \psi)$ is as in Proposition 3.3). We assume that $(F, \psi)$ is $(0, n)$-stable, and also assume that (4.1) holds.

Let $S_{x} \subset F_{x} / l_{x}$ be an isotropic subspace of dimension $n$. The isotropic subspace of dimension $n$ are parameterized by

$$
Y=\operatorname{Gr}_{\text {iso }, n}\left(F_{x} / l_{x}\right) \cong \operatorname{GO}(2 n, \mathbb{C}) / Q
$$


This subspace $S_{x}$ induces a commutative diagram

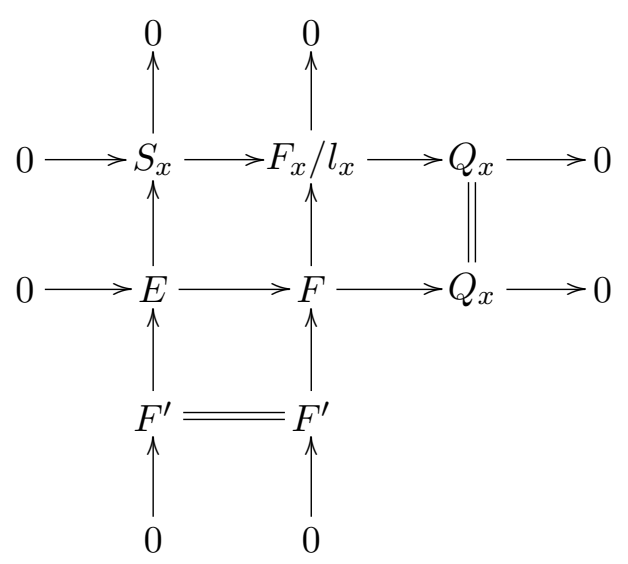

Note that this diagram is different from (4.2), because in the top row we have $F_{x} / l_{x}$ instead of $F_{x}$; in the bottom row, instead of $F(-x)$, we have a new vector bundle $F^{\prime}$. Arguing as in the even case, we finally obtain a commutative diagram on $Y$, with $\mathcal{S}$ and $\mathcal{Q}$ respectively being the universal isotropic subbundle and quotient of $F_{x} / l_{x}$

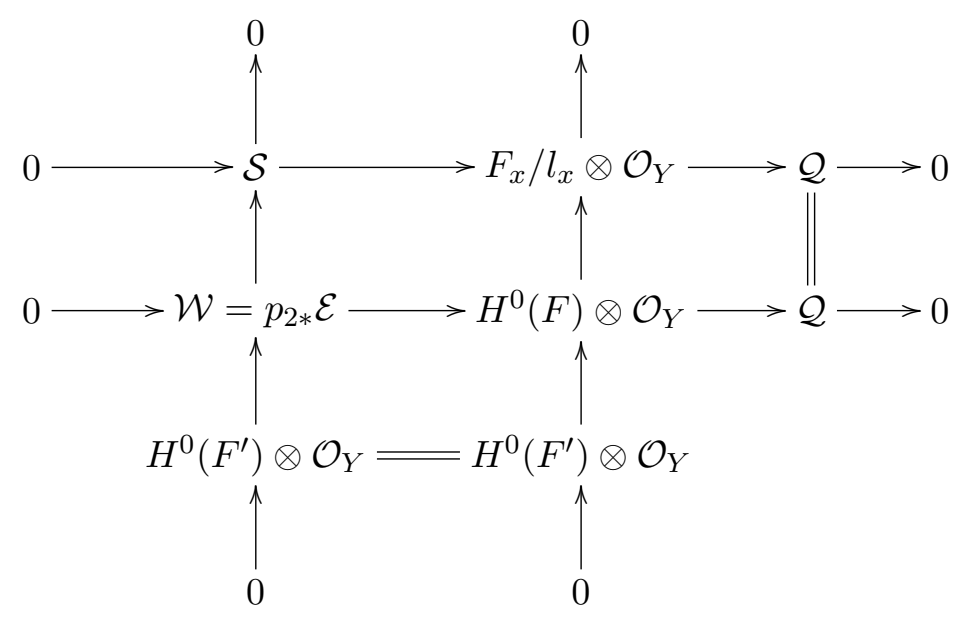

Proposition 4.1. Let $Q \subset \mathrm{GO}(2 n, \mathbb{C})$ be the maximal parabolic subgroup preserving a fixed isotropic subbundle $V \subset \mathbb{C}^{2 n}$ of dimension $n$. Then the Picard group of $Y=\operatorname{GO}(2 n, \mathbb{C}) / Q$ is $\mathbb{Z}$, the universal vector subbundle

$$
\mathcal{S} \longrightarrow \mathrm{GO}(2 n, \mathbb{C}) / Q
$$


is stable, and the determinant of $\mathcal{S}$ is $-2 A$, where $A$ is the ample generator of $\operatorname{Pic}(Y)$.

Proof. Note that

$$
\mathrm{GO}(2 n, \mathbb{C}) / Q=\mathrm{SO}(2 n, \mathbb{C}) / Q^{\prime}=\operatorname{Spin}(2 n, \mathbb{C}) / \widetilde{Q}^{\prime},
$$

where $Q^{\prime}=Q \bigcap \mathrm{SO}(2 n, \mathbb{C})$, and $\widetilde{Q}^{\prime}$ is the corresponding maximal parabolic subgroup in $\operatorname{Spin}(2 n, \mathbb{C})$. Therefore, $\widetilde{Q}^{\prime} \longrightarrow Q^{\prime}$ is a 2 -to-1 covering which restricts to $t \longmapsto t^{2}$ on the center.

The spin group is semisimple and simply connected, hence the Picard group of $\operatorname{Spin}(2 n, \mathbb{C}) / \widetilde{Q}^{\prime}$ is identified with the character group of $\widetilde{Q}^{\prime}$, which is equal to the character group of the Levi quotient $L\left(\widetilde{Q}^{\prime}\right)$; since $\widetilde{Q}^{\prime}$ is maximal, this character group is equal to $\mathbb{Z}$. Therefore, Pic $Y=\mathbb{Z}([6,7])$.

Take another isotropic subspace $W \subset \mathbb{C}^{2 n}$ such that $V+W=\mathbb{C}^{2 n}$. The orthogonal form on $\mathbb{C}^{2 n}$ identifies $W$ with $V^{\vee}$. A Levi subgroup $L\left(Q^{\prime}\right)$ of $Q^{\prime}$ is defined by all orthogonal automorphisms of $\mathbb{C}^{2 n}$ that preserve the direct sum decomposition $V \oplus W$, i.e., automorphisms taking $V$ to $V$ and $W$ to $W$.

Since $W=V^{\vee}$, the Levi subgroup $L\left(Q^{\prime}\right) \subset Q^{\prime}$ is identified with $\operatorname{GL}(V)$, sitting inside $\mathrm{SO}(2 n, \mathbb{C})$ as

$$
A \longmapsto\left(\begin{array}{cc}
A & 0 \\
0 & \left(A^{t}\right)^{-1}
\end{array}\right)
$$

using the above decomposition $\mathbb{C}^{2 n}=V \oplus V^{\vee}$. Consequently, the character $f$ of $L\left(Q^{\prime}\right)=\mathrm{GL}(V)$ defined by

$$
f(A) \longmapsto \operatorname{det} A,
$$

where $A$ is as in (4.8), generates the character group of $L\left(Q^{\prime}\right)$.

Since $\widetilde{Q}^{\prime} \longrightarrow Q^{\prime}$ is a 2-to- 1 covering, restricting to $t \longmapsto t^{2}$ on the center, the character $f$ corresponds to twice the generator of the character group of $L\left(\widetilde{Q}^{\prime}\right)$. The line bundle over $Y$ defined by the above character $f$ of $L\left(Q^{\prime}\right)$ coincides with the top exterior power of the tautological subbundle $\mathcal{S}$. Therefore, the first Chern class of the vector bundle $\mathcal{S}$ over the complete homogeneous space $Y$ is equal to twice a generator of the Picard group of $Y$. It is easy to see that $c_{1}(\mathcal{S})$ is nonpositive.

Since $\mathcal{S}$ is identified with the vector bundle associated to the principal $Q^{\prime}$-bundle over $Y$ for an irreducible representation of the Levi quotient of $Q^{\prime}$, a theorem due to Ramanan and Umemura says that the vector bundle $\mathcal{S}$ is stable (see [17, page 136, Theorem 2.4]). 
Corollary 4.1. The Hecke morphism $\Psi: Y \longrightarrow \mathcal{M}_{L}$ in (4.4) induces a nonzero homomorphism $\Psi^{*}: \operatorname{Pic} \mathcal{M}_{L} \longrightarrow \operatorname{Pic} Y$.

Proof. Since $(F, \psi)$ is $(0, n)$-stable, we obtain a family of stable orthogonal bundles $(E, \varphi)$, and the morphism $\Psi$ is well defined.

In the even case (respectively, odd case), it follows from (4.5) (respectively, (4.7)) that $\operatorname{deg} \mathcal{W}=\operatorname{deg} \mathcal{S}$, which by Proposition 4.1 is equal to -2 . But if the induced homomorphism $\Psi^{*}$ were zero, then we would have had $\operatorname{deg} \mathcal{W}=0$.

\section{Stability of Picard bundle}

Let

$$
\mathcal{M}_{L}^{0} \subset \mathcal{M}_{L}
$$

be the locus of stable principal bundles for which the automorphism group coincides with the center of $\mathrm{GO}(r, \mathbb{C})$.

Lemma 5.1. The subset $\mathcal{M}_{L}^{0}$ in (5.1) is Zariski open, and its complement is of codimension at least two.

Proof. Let $Z(\mathrm{GO}(r, \mathbb{C})) \subset \mathrm{GO}(r, \mathbb{C})$ be the center. Let $E_{\mathrm{GO}(r, \mathbb{C})} \in \mathcal{M}_{L}$ be a stable principal $\mathrm{GO}(r, \mathbb{C})$-bundle. Assume that the automorphism group $\operatorname{Aut}\left(E_{\mathrm{GO}(r, \mathbb{C})}\right)$ has an element $\tau$ which does not lie in $Z(\operatorname{GO}(r, \mathbb{C}))$. The Lie algebra of $\operatorname{Aut}\left(E_{\mathrm{GO}(r, \mathbb{C})}\right)$ coincides with the global section $H^{0}\left(X, E_{\mathrm{GO}(r, \mathbb{C})}\right.$ $(\mathfrak{g} \mathfrak{o}(r, \mathbb{C})))$ of the adjoint vector bundle, and hence it coincides with the center $\mathfrak{z}(\mathfrak{g o}(r, \mathbb{C}))$ because $E_{\mathrm{GO}(r, \mathbb{C})}$ is stable. Therefore, the quotient Aut $\left(E_{\mathrm{GO}(r, \mathbb{C})}\right) / Z(\mathrm{GO}(r, \mathbb{C}))$ is a finite group. This implies that $\tau$ is a semisimple element.

Since $\tau$ is semisimple, it defines a conjugacy class of elements of $\operatorname{GO}(r, \mathbb{C})$ (see the second paragraph of $[2$, Section 3$])$. Fix an element $\bar{\tau} \in \operatorname{GO}(r, \mathbb{C})$ in the conjugacy class given by $\tau$. Let

$$
\mathcal{C}_{\bar{\tau}} \subset \mathrm{GO}(r, \mathbb{C})
$$

be the centralizer of $\bar{\tau}$. The principal $\mathrm{GO}(r, \mathbb{C})$-bundle $E_{\mathrm{GO}(r, \mathbb{C})}$ admits a holomorphic reduction of structure group to $\mathcal{C}_{\bar{\tau}}[2$, p. 230, Theorem 3.2]. Since the principal $\mathrm{GO}(r, \mathbb{C})$-bundle $E_{\mathrm{GO}(r, \mathbb{C})}$ is stable, it does not admit any reduction of structure group to any Levi subgroup of some proper parabolic 
subgroup of $\operatorname{GO}(r, \mathbb{C})$. Hence $\mathcal{C}_{\bar{\tau}}$ is not a Levi subgroup of some proper parabolic subgroup of $\mathrm{GO}(r, \mathbb{C})$.

Up to conjugacy, there are only finitely many semisimple elements

$$
c_{1}^{\prime}, \ldots, c_{m}^{\prime} \in \operatorname{GO}(r, \mathbb{C}) / Z(\operatorname{GO}(r, \mathbb{C}))
$$

whose centralizer is not a Levi subgroup of some parabolic subgroup of the semisimple group $\operatorname{GO}(r, \mathbb{C}) / Z(\mathrm{GO}(r, \mathbb{C}))$ (see $[9$, p. 113]). Fix elements

$$
c_{1}, \ldots, c_{m} \in \operatorname{GO}(r, \mathbb{C})
$$

such that $c_{i}$ projects to $c_{i}^{\prime}$.

Let

$$
\mathcal{C}_{c_{i}} \subset \mathrm{GO}(r, \mathbb{C})
$$

be the centralizer of $c_{i}$. Let $\mathcal{M}\left(\mathcal{C}_{c_{i}}\right)$ be the moduli space of stable principal $\mathcal{C}_{c_{i}}$-bundles over $X$ that maps to $\mathcal{M}_{L}$ by extension of structure group of principal $\mathcal{C}_{c_{i}}$-bundles to $\operatorname{GO}(r, \mathbb{C})$. Note that the complement $\mathcal{M}_{L} \backslash \mathcal{M}_{L}^{0}$ is the image of the union $\bigcup_{i} \mathcal{M}\left(\mathcal{C}_{c_{i}}\right)$. From the formula for dimension of a moduli space of principal bundles it follows immediately that

$$
\operatorname{dim} \mathcal{M}\left(\mathcal{C}_{c_{i}}\right) \leq \operatorname{dim} \mathcal{M}_{L}-2
$$

This completes the proof of the lemma.

The projectivized Picard bundle is a principal $\operatorname{PGL}(N, \mathbb{C})$-bundle $P^{\mathrm{PGL}}$ on $\mathcal{M}_{L}^{0}$, such that for any point $(E, \varphi) \in \mathcal{M}_{L}^{0}$, the fiber over $(E, \varphi)$ of the associated projective bundle $P^{\mathrm{PGL}}\left(\mathbb{P}^{N-1}\right)$ is canonically identified with $\mathbb{P}\left(H^{0}(X, E)\right)$. Note that $N=\operatorname{dim} H^{0}(X, E)$. From the construction of the moduli space it follows that the projectivized Picard bundle exists.

The moduli space $\mathcal{M}_{L}$ has a natural compactification, namely the coarse moduli space of semistable orthogonal bundles, which known to be a normal projective variety. The complement $\overline{\mathcal{M}}_{L} \subset \mathcal{M}_{L}$ has codimension at least two, and hence we can think of the projectivized Picard bundle as a rational principal bundle (their definition is recalled below) on the projective variety $\overline{\mathcal{M}}_{L}$.

Recall from [14] that a rational principal bundle on a normal projective variety $M$ is a principal bundle $P$ on a big open set $U \subset M$ (i.e., an open set whose complement has codimension at least two). A rational principal bundle is said to be stable (respectively, semistable) with respect to a 
polarization $\mathcal{O}_{M}(1)$ if for every reduction $\left.P^{Q} \subset P\right|_{V}$ to a maximal parabolic subgroup $Q$ defined on big open subset $V \subset U$, and for all nontrivial dominant characters of $Q$ which are trivial on the center of $G$, the inequality $\operatorname{deg} P^{Q}(\chi)<0$ (respectively, $\operatorname{deg} P^{Q}(\chi) \leq 0$ ) holds, where

$$
P^{Q}(\chi)=\left(P^{Q} \times \mathbb{C}_{\chi}\right) / Q
$$

is the line bundle over $V$ associated to $P^{Q}$ for the character $\chi$, and the degree is calculated with respect to the polarization $\mathcal{O}_{M}(1)$.

Proposition 5.1. Assume that $g(X)>n$ where $n=[r / 2]$. Fix distinct points $x_{1}, \ldots, x_{m} \in X$. Let $Z$ be a subscheme of $\mathcal{M}_{L}$ of codimension at least two. Then there is a nonempty Zariski open subset $V_{0} \subset \mathcal{M}_{L}^{0} \subset \mathcal{M}_{L}$ such that the following two hold:

(1) If $(E, \varphi) \in V_{0}$, is $(n, n)$-stable.

(2) Take any $(E, \varphi) \in V_{0}$ and choose a point $x_{i}$. Let $(F, \psi)$ be a general Hecke transformation of $(E, \varphi)$ with respect to $x_{i}$. If $\Psi: Y \longrightarrow \mathcal{M}_{L}$ is the Hecke morphism, then $\Psi^{-1}(Z)$ is either empty or its codimension in $Y$ is at least two.

Proof. We will first assume that $m=1$, so there is only one point $x_{1}=x$. If $m>1$, we take the intersection of the open subsets of $\mathcal{M}_{L}^{0}$ corresponding to each point $x_{i}$.

Let $\mathcal{M}_{L}^{(n, n)} \subset \mathcal{M}_{L}^{0}$ be the open subset of $(n, n)$-stable orthogonal bundles. This is dense because of Proposition 3.5.

Let $p: \mathcal{Y} \longrightarrow \mathcal{M}_{L}^{(n, n)}$ be the fibration whose fiber over any $(E, \varphi)$ is canonically isomorphic to $\operatorname{Gr}_{\text {iso, } n}\left(E_{x}^{\vee}\right)$, i.e., the set of isotropic subspaces of $E_{x}^{\vee}$ of dimension $n$. This fibration can easily be constructed because all the points in $\mathcal{M}_{L}^{(n, n)} \subset \mathcal{M}_{L}^{0}$, by definition, correspond to orthogonal bundles whose automorphisms are scalars. Therefore, by Proposition 3.2 or Proposition 3.4 , each point of $\mathcal{Y}$ corresponds to a short exact sequence

$$
0 \longrightarrow E \longrightarrow F \longrightarrow Q_{x} \longrightarrow 0
$$

where $F$ is the corresponding Hecke transform; indeed, if we apply Hom $\left(\cdot, \mathcal{O}_{X}\right)$ to $(3.3)$ or $(3.7)$ we obtain this exact sequence).

Let $q: \mathcal{T} \longrightarrow \mathcal{Y}$ be the fibration whose fiber over a point corresponding to a short exact sequence as in (5.2) is canonically isomorphic to $\mathrm{Gr}_{\text {iso, } n}$ $\left(F_{x} / l_{x}\right)$ if $r$ is odd, or canonically isomorphic to $\operatorname{Gr}_{\text {iso }, n}\left(F_{x}\right)$ if $r$ is even. 
Therefore, each point of $\mathcal{T}$ corresponds to a diagram

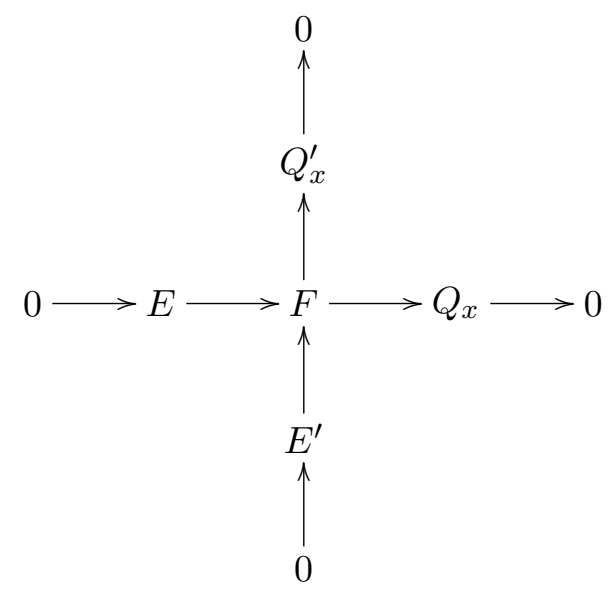

For all points in $\mathcal{T}$, the orthogonal bundle $(E, \varphi)$ is $(n, n)$-stable, hence $(F, \psi)$ is $(0, n)$-stable, and then $\left(E^{\prime}, \varphi^{\prime}\right)$ is stable as an orthogonal bundle. Therefore, by sending a point corresponding to a diagram in (5.3) to $\left(E^{\prime}, \varphi^{\prime}\right)$, we obtain a morphism $p^{\prime}: \mathcal{T} \longrightarrow \mathcal{M}_{L}$. So,

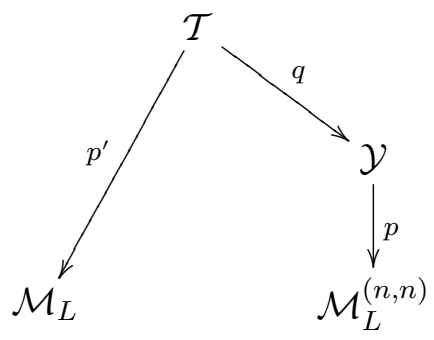

Note that, for each point $y \in \mathcal{Y}$, the fiber $q^{-1}(y)$ is identified with a Hecke cycle $Y$ which is mapped by $p^{\prime}$ to $\mathcal{M}_{L}$.

Let $Z^{\prime}=p^{\prime-1}(Z)$. If $q\left(Z^{\prime}\right)$ is not dense in $\mathcal{Y}$, take an open subset in the complement; the image of this open set under $p$ satisfies the condition of the proposition.

It remains to analyze the case when $q\left(Z^{\prime}\right)$ is dense in $\mathcal{Y}$. In this case, the dimension of the generic fiber of $\left.q\right|_{Z^{\prime}}$ is

$$
\begin{aligned}
\operatorname{dim} Z^{\prime}-\operatorname{dim} \mathcal{Y}= & \operatorname{dim} Z+\operatorname{dim} p^{\prime}-\operatorname{dim} \mathcal{Y}=\operatorname{dim} Z+\operatorname{dim} p^{\prime}-\operatorname{dim} \mathcal{M}_{L}^{(n, n)} \\
& -\operatorname{dim} p=\left(\operatorname{dim} p-\operatorname{dim} p^{\prime}\right)+\left(\operatorname{dim} Z-\operatorname{dim} \mathcal{M}_{L}\right) \\
\leq & \operatorname{dim} Y-2 .
\end{aligned}
$$

This completes the proof of the proposition. 
We have $\operatorname{Pic}\left(\mathcal{M}_{L}^{0}\right)=\operatorname{Pic}\left(\mathcal{M}_{L}\right)=\mathbb{Z}$ (see $\left.[1,5]\right)$, so $\mathcal{M}_{L}^{0}$ has a unique polarization.

We can now state and prove the main theorem. Let $P^{\mathrm{PGL}}$ be the above defined projectivized Picard bundle on the moduli space $\mathcal{M}_{L}^{0}$ of stable orthogonal bundles

$$
(E, \varphi: E \otimes E \longrightarrow L)
$$

on $X$. Denote $r=\operatorname{rk} E=r$, and $d=\operatorname{deg} E$.

Theorem 5.1. Assume that $g(X)>n$ where $n=[r / 2]$. Also assume that $d>(2 g-2) r+r$. Then, the projectivized Picard bundle $P^{\mathrm{PGL}}$ over the moduli space $\mathcal{M}_{L}^{0}$ is stable (since the Picard group of $\mathcal{M}_{L}^{0}$ is $\mathbb{Z}$, stability is independent of choice of polarization).

Proof. Let

$$
P^{\bar{Q}} \subset P^{\mathrm{PGL}}
$$

be a reduction of structure group of $P$, on a big Zariski open set $U \subset \mathcal{M}_{L}^{0}$, to a maximal parabolic subgroup $\bar{Q} \subset \operatorname{PGL}(N, \mathbb{C})$ (recall that by a big Zariski open subset we mean one whose complement is of codimension at least two). The parabolic subgroup $\bar{Q}$ is the image of a unique maximal parabolic subgroup $Q$ of $\operatorname{GL}(N, \mathbb{C})$ by the natural projection $\operatorname{GL}(N, \mathbb{C}) \longrightarrow \operatorname{PGL}(N, \mathbb{C})$. We need to prove that for a nontrivial dominant character $\chi$ of $\bar{Q}$, the inequality

$$
\operatorname{deg}\left(P^{\bar{Q}}(\chi)\right)<0
$$

holds. If $A$ is the unique proper nonzero subspace of $\mathbb{C}^{N}$ preserved by $Q$, then any nontrivial dominant character of $\bar{Q}$ is a positive multiple of the character defined by the natural action of $\bar{Q}$ on the line $\bigwedge^{\text {top }} \operatorname{Hom}\left(\mathbb{C}^{N} / A, A\right)$.

The strategy of the proof is to use a Hecke morphism $\Psi: Y \longrightarrow \mathcal{M}_{L}$ (defined in $(4.4)$ ). We denote the restriction to $Y^{0}:=\Psi^{-1}\left(\mathcal{M}_{L}^{0}\right)$ as

$$
\Psi^{0}: Y^{0} \longrightarrow \mathcal{M}_{L}^{0}
$$

Then we calculate the degree using the pullback of $P^{\bar{Q}}$ to an open subset of $Y^{0}$. For this to work, we need that the Hecke cycle be "general enough" in the sense that $\Psi^{-1}\left(\mathcal{M}_{L} \backslash U\right)$ has codimension at least two. This is to ensure that the inclusion map $Y^{0} \hookrightarrow Y$ induces an isomorphism of Picard groups. 
Fix $m$ distinct points $x_{1}, \ldots, x_{m} \in X$ with $m>\operatorname{deg}(E)(1+1 / \operatorname{rk}(E))$. Set

$$
Z=U^{c}=\mathcal{M}_{L} \backslash U
$$

(Recall that the Picard bundle is defined on $\mathcal{M}_{L}^{0} \subset \mathcal{M}_{L}$, and the reduction $P^{\bar{Q}}$ is defined on $U \subset \mathcal{M}_{L}^{0}$.) Fix an orthogonal bundle $(E, \varphi)$ over $X$ corresponding to a point in the intersection of $U$ with the open subset of $\mathcal{M}_{L}^{0}$ given by Proposition 5.1.

The reduction of structure group $P^{\bar{Q}}$ gives a projective subbundle

$$
P^{\bar{Q}}\left(\mathbb{P}^{N^{\prime}-1}\right) \subset P^{\mathrm{PGL}}\left(\mathbb{P}^{N-1}\right)
$$

over $U$. The fiber of $P^{\mathrm{PGL}}\left(\mathbb{P}^{N-1}\right)$ over $(E, \varphi)$ is canonically isomorphic to $\mathbb{P}\left(H^{0}(E)\right)$, and the fiber of $P^{\bar{Q}}\left(\mathbb{P}^{N^{\prime}-1}\right)$ defines a subspace $V^{\prime} \subset H^{0}(E)$.

Fix a nonzero element

$$
s \in V^{\prime} \subset H^{0}(X, E),
$$

which we are going to consider as a section of $E$.

We claim that the above section $s$ of $E$ cannot vanish over more than $\operatorname{deg}(E) / \operatorname{rk}(E)$ points. Indeed, if $D$ is a subset of $\left\{x_{1}, \ldots, x_{m}\right\}$, and $s$ vanishes in all points of $D$, then the section $s: \mathcal{O}_{X} \longrightarrow E$ factors through $\mathcal{O}_{X}(D)$, and the semistability of $E$ (Proposition 2.2) implies that $\operatorname{deg}(D) \leq$ $\operatorname{deg}(E) / \operatorname{rk}(E)$.

Analogously, if $E^{V^{\prime}}$ is the coherent subsheaf of $E$ generated by the sections $V^{\prime} \subset H^{0}(X, E)$, then $E^{V^{\prime}}$ cannot fail to be a subbundle over more than $\operatorname{deg}(E)$ points. Indeed, if $D$ is a subset of $\left\{x_{1}, \ldots, x_{m}\right\}$, and $E^{V^{\prime}}$ is not a subbundle on all points of $D$, then the inclusion $E^{V^{\prime}} \subset E$ factors through a subsheaf $\widetilde{E} \subset E$ with

$$
\operatorname{deg}(\widetilde{E}) \geq \operatorname{deg}\left(E^{V^{\prime}}\right)+\operatorname{deg} D
$$

Since $E^{V^{\prime}}$ is generated by global sections, it follows that $\operatorname{deg}\left(E^{V^{\prime}}\right) \geq 0$. On the other hand, the stability condition of $E$ implies that

$$
\operatorname{deg} \widetilde{E} \leq \operatorname{deg}(E) \operatorname{rk}(\widetilde{E}) / \operatorname{rk}(E) \leq \operatorname{deg}(E),
$$

therefore $\operatorname{deg}(D) \leq \operatorname{deg}(E)$.

Consequently, we can choose a point $x \in\left\{x_{1}, \ldots, x_{m}\right\}$ such that $s(x) \neq 0$, the sheaf $E^{V^{\prime}}$ generated by $V^{\prime}$ is locally free at $x$, and the induced homomorphism

$$
E_{x}^{V^{\prime}} \longrightarrow E_{x}
$$

is injective. 
If $r=2 n$, using Proposition 5.1, we can choose an isotropic subspace $S_{x} \subset E_{x}$ of dimension $n$ such that

$$
s(x) \notin S_{x},
$$

and the subscheme $\Psi^{-1}(Z) \subset Y$ is either empty or it is of codimension at least two in $Y=\operatorname{Gr}_{\text {iso }, n}\left(F_{x}\right)$, where $\Psi: Y \longrightarrow \mathcal{M}_{L}$ is the Hecke morphism associated to $(F, \psi)$ and the point $x \in X$ (see (4.4)).

If $r=2 n+1$, using again Proposition 5.1, we choose an isotropic subspace $W_{x} \subset E_{x}$ of dimension $n$ such that $s(x) \notin W_{x}^{\perp}$.

This bound on the codimension of $\Psi^{-1}(Z)$ implies that $\operatorname{Pic}(Y)=\operatorname{Pic}\left(Y_{U}\right)$, where $Y_{U}:=Y \backslash \Psi^{-1}(Z)$ is the complement. Now using Corollary 4.1 it follows that the homomorphism

$$
\Psi_{U}^{*}: \operatorname{Pic}\left(\mathcal{M}_{L}\right) \longrightarrow \operatorname{Pic}\left(Y_{U}\right)=\operatorname{Pic}(Y)
$$

is nonzero. Therefore, there is a positive rational number $k$ such that

$$
\operatorname{deg}\left(P^{\bar{Q}}(\chi)\right)=k \cdot \operatorname{deg}\left(\Psi_{U}^{*} P^{\bar{Q}}(\chi)\right)
$$

Let $\mathcal{E}$ be the family of Hecke transformations parameterized by $Y$ (see (4.3)). The pulled back projective bundle $\Psi^{*} P^{\mathrm{PGL}}$ on $Y$ lifts to the principal $\mathrm{GL}(N, \mathbb{C})$-bundle $P^{\mathrm{GL}}$ over $Y$ associated to

$$
\mathcal{W}=p_{2 *} \mathcal{E}
$$

where $p_{2}: X \times Y \longrightarrow Y$ is the natural projection. This means that there is an isomorphism

$$
\mathbb{P}(\mathcal{W}) \cong P^{\mathrm{PGL}}\left(\mathbb{P}^{N-1}\right)
$$

Let $\Psi_{U}: Y_{U} \longrightarrow U$ be the restriction of $\Psi$ to $Y_{U}=\Psi^{-1}(U)$. We claim that the pullback $\Psi_{U}^{*} P^{\bar{Q}}$ of the reduction in (5.4) is given by a subbundle of $\mathcal{W}$. In other words, there is a subbundle

$$
\mathcal{H} \hookrightarrow \mathcal{W}
$$

on $Y_{U}$ such that the subbundle $\mathbb{P}(\mathcal{H}) \subset \mathbb{P}(\mathcal{W})$ is identified with the subbundle $P^{\bar{Q}}\left(\mathbb{P}^{N^{\prime}-1}\right) \subset P^{\bar{Q}}\left(\mathbb{P}^{N-1}\right)$ by the isomorphism in $(5.9)$.

To prove the above claim, note that, since $\Psi_{U}^{*} P^{\bar{Q}}$ is a reduction of structure group of $\Psi_{U}^{*} P^{\mathrm{PGL}}$, it is given by a section of $\Psi_{U}^{*} P^{\mathrm{PGL}}(\operatorname{PGL}(N, \mathbb{C}) / \bar{Q})$. 
But

$$
\Psi_{U}^{*} P^{\mathrm{PGL}}(\operatorname{PGL}(N, \mathbb{C}) / \bar{Q})=\Psi_{U}^{*} P^{\mathrm{GL}}(\mathrm{GL}(N, \mathbb{C}) / Q) .
$$

Hence such a section gives a reduction of structure group to $Q \subset \operatorname{GL}(N, \mathbb{C})$ of the principal $\operatorname{GL}(N, \mathbb{C})$-bundle associated to $\mathcal{W}$. Since this is equivalent to giving a subbundle $\mathcal{H} \hookrightarrow \mathcal{W}$, the above claim, that the pullback $\Psi_{U}^{*} P^{\bar{Q}}$ of the reduction in (5.4) is given by a subbundle of $\mathcal{W}$, is proved.

If $\chi$ is a dominant character of $\bar{Q}$, then it is easy to check that

$$
\operatorname{deg}\left(\Psi_{U}^{*} P^{\bar{Q}}(\chi)\right)=k^{\prime}(\operatorname{rk}(\mathcal{W}) \operatorname{deg}(\mathcal{H})-\operatorname{deg}(\mathcal{W}) \operatorname{rk}(\mathcal{H}))
$$

for some positive number $k^{\prime}$. Indeed, this follows from the earlier remark that any nontrivial dominant character of $\bar{Q}$ is a positive multiple of the character defined by the natural action of $\bar{Q}$ on the line $\bigwedge^{\text {top }} \operatorname{Hom}\left(\mathbb{C}^{N} / A, A\right)$.

In view of (5.11), (5.8) and (5.5), to prove the theorem it is enough to check that $\mathcal{H}$ does not contradict the stability of $\mathcal{W}$, meaning

$$
\operatorname{rk}(\mathcal{W}) \operatorname{deg}(\mathcal{H})-\operatorname{deg}(\mathcal{W}) \operatorname{rk}(\mathcal{H})<0
$$

If $r$ is even, note that since $S_{x}$ is the image of $F(-x)_{x}$ in $E_{x}$ in diagram (4.2), it follows that $s \notin H^{0}(F(-x)$ ) (see (5.7)).

If $r$ is odd, then the vector space $S_{x}$ is the image of $F_{x}^{\prime}$ in $E_{x}$ in diagram (4.6), and it follows that $s \notin H^{0}\left(F^{\prime}\right)$.

In both cases, using this and (4.5) (respectively, (4.7)) for the even (respectively, odd) case we conclude that the composition

$$
\mathcal{H} \hookrightarrow \mathcal{W} \longrightarrow \mathcal{S}
$$

has nonzero image.

To unify the notation for the even and odd cases, denote

$$
F_{0}:=F(-x)
$$

if $r=2 n$, and

$$
F_{0}:=F^{\prime}
$$

if $r=2 n+1$. Consider the commutative diagram

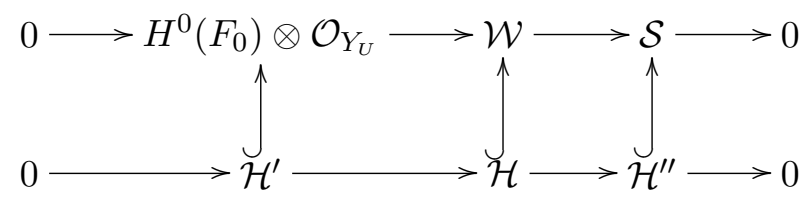


We have seen that $\mathcal{H}^{\prime \prime} \neq 0$. The vector bundle $\mathcal{S}$ is stable of degree -2 (Proposition 4.1). We have $\operatorname{deg} \mathcal{H}^{\prime \prime} \leq-1$ because $\mathcal{H}^{\prime \prime}$ is a subsheaf of a stable vector bundle of negative degree, and also $\operatorname{deg} \mathcal{H}^{\prime} \leq 0$ as it is a subsheaf of a semistable vector bundle of degree zero; see (5.14). If $\operatorname{deg} \mathcal{H}^{\prime \prime} \leq-2$, then $\operatorname{deg} \mathcal{H} \leq-2$, and hence (5.12) holds.

Therefore, for the rest of the proof we consider the case $\operatorname{deg} \mathcal{H}^{\prime \prime}=-1$.

Assume that (5.12) does not hold, in other words, assume that

$$
\operatorname{rk} \mathcal{H} \geq \frac{h^{0}(E)}{2} .
$$

We have rk $\mathcal{H}^{\prime \prime}<\operatorname{rk} \mathcal{S}$, because if we had equality we should have

$$
\operatorname{deg} \mathcal{H}^{\prime \prime} \leq \operatorname{deg} \mathcal{S}=-2
$$

but we are now in the case $\operatorname{deg} \mathcal{H}^{\prime \prime}=-1$. Hence the stability condition of $\mathcal{S}$ implies that

$$
\operatorname{rk} \mathcal{H}^{\prime \prime}<\frac{n}{2}
$$

By our choice of $(E, \varphi) \in \mathcal{M}_{L}^{0}$, the coherent sheaf $\mathcal{H}$ is locally free, and the induced homomorphism $\mathcal{H}_{(E, \varphi)} \longrightarrow \mathcal{W}_{(E, \varphi)}$ is injective. Let $V^{\prime}=$ $\mathcal{H}_{(E, \varphi)} \subset \mathcal{W}_{(E, \varphi)}=H^{0}(E)$. By Lemma 5.2, Lemma 2.2 and (5.15),

$$
\operatorname{rk} E^{V^{\prime}} \geq \operatorname{rk} E \frac{\operatorname{dim} V^{\prime}}{h^{0}(E)}=r \frac{\operatorname{rk} \mathcal{H}}{h^{0}(E)} \geq \frac{r}{2} .
$$

By our choice of $x \in X$, the induced homomorphism $E_{x}^{V^{\prime}} \longrightarrow E_{x}$ is injective (see (5.6)). Restricting the commutative diagram (5.14) to the fiber over $(E, \varphi)$, we obtain the following commutative diagram

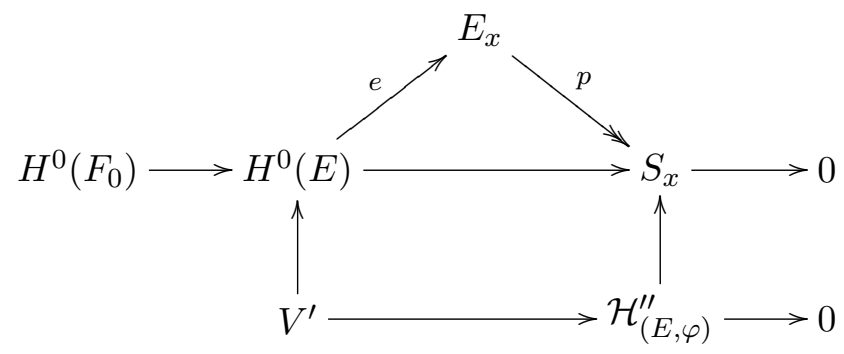

where $e$ is the evaluation morphism and the projection $p$ fits in an exact sequence

$$
0 \longrightarrow N_{x} \longrightarrow E_{x} \stackrel{p}{\longrightarrow} S_{x} \longrightarrow 0 .
$$


It follows that

$$
\begin{aligned}
\mathcal{H}_{(E, \varphi)}^{\prime \prime} & =\operatorname{Im}\left(V^{\prime} \rightarrow S_{x}\right)=\operatorname{Im}\left(V^{\prime} \hookrightarrow H^{0}(E) \rightarrow E_{x} \rightarrow S_{x}\right) \\
& =\operatorname{Im}\left(E_{x}^{V^{\prime}} \hookrightarrow E_{x} \rightarrow S_{x}\right) \cong \frac{E_{x}^{V^{\prime}}}{E_{x}^{V^{\prime}} \cap N_{x}}
\end{aligned}
$$

It is easy to check that

$$
\operatorname{dim} \frac{E_{x}^{V^{\prime}}}{E_{x}^{V^{\prime}} \cap N_{x}} \leq \operatorname{dim} S_{x},
$$

with equality holding if $N_{x}$ is a general subspace of $E_{x}$.

On the other hand, $\operatorname{dim} S_{x}=n$, and therefore, for a general $N_{x} \subset E_{x}$, we have $\mathrm{rk} \mathcal{H}^{\prime \prime}=n$, which contradicts (5.16). This implies that the assumption in (5.15) is false, meaning (5.12) holds, and the theorem if proved.

Lemma 5.2. Let $E$ be a semistable vector bundle on $X$ of degree $d$ and rank $r$. If $d>(2 g-2) r+r$, then for all subspaces $V^{\prime} \subset H^{0}(E)$,

$$
r \operatorname{dim} V^{\prime}-\operatorname{rk} E^{V^{\prime}} h^{0}(E) \leq 0,
$$

where $E^{V^{\prime}} \subset E$ is the subsheaf generated by $V^{\prime} \subset H^{0}(E)$.

Proof. Let $E^{\prime}$ be a semistable vector bundle of degree $d^{\prime}$ and rank $r^{\prime}$. If $d^{\prime}>(2 g-2) r^{\prime}$, then

$$
h^{0}\left(E^{\prime}\right)=d^{\prime}-r^{\prime}(g-1)
$$

because $h^{1}\left(E^{\prime}\right)=h^{0}\left(K_{X} \otimes E^{\prime \vee}\right)$ (Serre duality), and this is zero since $K_{X} \otimes$ $E^{\prime \vee}$ is a semistable vector bundle of negative degree. On the other hand, if $0 \leq d^{\prime} \leq(2 g-2) r^{\prime}$, then by Clifford's theorem (see, for instance, [8, Theorem 2.1]),

$$
h^{0}\left(E^{\prime}\right) \leq \frac{d^{\prime}}{2}+r^{\prime}
$$

Therefore, if $h^{0}\left(E^{\prime}\right)>(g-1) r^{\prime}+r^{\prime}$, then we must be in the first case, and hence

$$
d^{\prime}=h^{0}\left(E^{\prime}\right)+r^{\prime}(g-1)>2 r^{\prime}(g-1)+r^{\prime}
$$

if $h^{0}\left(E^{\prime}\right)>(g-1) r^{\prime}+r^{\prime}$. 
Let $E^{i}, 1 \leq i \leq \ell$, be the successive quotients of the Harder-Narasimhan filtration of $E^{V^{\prime}}$. We have

$$
\operatorname{dim} V^{\prime} \leq h^{0}\left(E^{V^{\prime}}\right) \leq \sum h^{0}\left(E^{i}\right),
$$

for all $i$. Denote $r_{i}=\operatorname{rk} E^{i}$. If $h^{0}\left(E^{i}\right) \leq(g-1) r_{i}+r_{i}$, then applying (5.18) to $E$, we have

$$
r \cdot h^{0}\left(E^{i}\right)-r_{i} \cdot h^{0}(E)<r\left(r_{i}(g-1)+r_{i}\right)-r_{i}(r(g-1)+r) \leq 0 .
$$

On the other hand, if $h^{0}\left(E^{i}\right)>(g-1) r_{i}+r_{i}$, then applying (5.20) and (5.18) to $E^{i}$ and $E$ we have

$$
r \cdot h^{0}\left(E^{i}\right)-r_{i} \cdot h^{0}(E)=r\left(d^{i}-r_{i}(g-1)\right)-r_{i}(d-r(g-1)) \leq 0,
$$

by the stability condition of $E$. Therefore

$$
r \operatorname{dim} V^{\prime}-\operatorname{rk} E^{V^{i}} h^{0}(E) \leq \sum\left(r h^{0}\left(E^{i}\right)-r_{i} h^{0}(E)\right) \leq 0 .
$$

This completes the proof of the lemma.

For a fixed holomorphic line bundle $\xi$ on $X$, sending any orthogonal bundle $\varphi: E \otimes E \longrightarrow L$ to the orthogonal bundle $(E \otimes \xi) \otimes(E \otimes \xi) \longrightarrow$ $L \otimes \xi^{\otimes 2}$ given by $\varphi$ we obtain an isomorphism between the corresponding moduli spaces. The pull-back of the projective Picard bundle under this isomorphism is the $\xi$-twisted projective Picard bundle, namely the projective bundle whose fiber over a point corresponding to $(E, \varphi)$ is canonically isomorphic to $\mathbb{P}\left(H^{0}(E \otimes \xi)\right)$. Using this isomorphism, we have the following corollary.

Corollary 5.1. Assume that $g(X)>n$ where $n=[r / 2]$. Let $\xi$ be a holomorphic line bundle of degree $m$. Assume also $d+r m>(2 g-2) r+r$. Then, the $\xi$-twisted projectivized Picard bundle $P^{\mathrm{PGL}}$ over the moduli space $\mathcal{M}_{L}^{0}$ is stable with respect to the unique polarization of $\mathcal{M}_{L}^{0}$.

In [4], we introduced Hecke transform for symplectic bundles. In the symplectic case we started with a $(0, n)$-stable symplectic bundle, performed a Hecke transform with an isotropic subspace of dimension $n$, and obtained a stable symplectic bundle. 
In the odd orthogonal case, i.e., when the group is $\operatorname{GO}(2 n+1, \mathbb{C})$, we cannot choose a subspace of half dimension. To obtain an orthogonal bundle in this case, we have to start with a singular bilinear form $(F, F \otimes F \longrightarrow M)$ (Proposition 3.3), which does not come from a principal bundle. The naive approach would be to start with a $(0, n)$-stable bundle as in Proposition 3.3 to obtain an orthogonal bundle. The difficulty is that not much is known about the properties of the moduli space of bilinear forms with singularities like this (neither smoothness nor dimension is known), and in particular, we do not know if the set of $(0, n)$-stable bundles is dense.

The strategy is then to perform two Hecke transforms instead of just one: we start with an $(n, n)$-stable orthogonal bundle. A Hecke transform with respect to a subspace of dimension $n$ will produce a singular (in the odd case) bilinear bundle $(F, F \otimes F \longrightarrow M)$, which is $(0, n)$-stable (Proposition 3.4), and then we perform a second Hecke transform (see Proposition 3.3) to get a stable orthogonal bundle. Therefore, we have to prove that the set of $(n, n)$-stable orthogonal bundles is nonempty (Proposition 3.5).

Another difficulty in the orthogonal case is that the determinant of the universal vector subbundle $\mathcal{S}$ on the Grassmannian parameterizing rank $n$ isotropic subspaces in $\mathbb{C}^{2 n}$ (endowed with the standard orthogonal form) is not a primitive element of the Picard group (see Proposition 4.1). Because of this, the proof of Theorem 5.1 is longer than in the symplectic case, where the determinant of the analogous universal bundle is a generator of the Picard group.

\section{Acknowledgment}

Research supported by the Spanish Ministerio de Educación y Ciencia [MTM 2007-63582].

\section{References}

[1] A. Beauville, Y. Laszlo and C. Sorger, The Picard group of the moduli of $G$-bundles on a curve, Compos. Math. 112 (1998), 183-216.

[2] V. Balaji, I. Biswas and D.S. Nagaraj, Krull-Schmidt reduction for principal bundles, J. Reine Angew. Math. 578 (2005), 225-234.

[3] I. Biswas, L. Brambila-Paz, T.L. Gómez and P.E. Newstead, Stability of the Picard Bundle, Bull. Lond. Math. Soc. 34 (2002), 561-568. 
[4] I. Biswas and T. Gómez, Hecke correspondence for symplectic bundles with application to the Picard bundles, Internat. J. Math. 17 (2006), 45-63.

[5] I. Biswas and N. Hoffmann, The line bundles on moduli stacks of principal bundles on a curve, Documenta Math. 15 (2010), 35-72.

[6] A. Borel and F. Hirzebruch, Characteristic classes and homogeneous spaces, I, Amer. J. Math. 80 (1958), 458-538.

[7] A. Borel and F. Hirzebruch, Characteristic classes and homogeneous spaces, II, Amer. J. Math. 81 (1959), 315-382.

[8] L. Brambila-Paz, I. Grzegorczyk, and P.E. Newstead, Geography of Brill-Noether loci for small slopes, J. Alg. Geom. 6 (1997), 645-669.

[9] F. Digne and J. Michel, Representations of finite groups of Lie type, London Math. Soc. Study Texts 21, Cambridge University Press, Cambridge, 1991.

[10] M.S. Narasimhan and S. Ramanan, Deformations of the moduli space of vector bundles over an algebraic curve, Ann. Math. 101 (1975), 391-417.

[11] M.S. Narasimhan and S. Ramanan, Geometry of Hecke cycles I, in: C. P. Ramanujan - A Tribute, Studies in Math. 8, T.I.F.R., Bombay, 1978, 291-345.

[12] S. Ramanan and A. Ramanathan, Some remarks on the instability flag, Tôhoku Math. J. 36 (1984), 269-291.

[13] A. Ramanathan, Stable principal bundles on a compact Riemann surface, Math. Ann. 213 (1975), 129-152.

[14] A. Ramanathan, Moduli for principal bundles, Algebraic geometry Proc. Summer Meeting, Univ. Copenhagen, Copenhagen, 1978, Lecture Notes in Math. 732, Springer, Berlin, 1979, 527-533.

[15] A. Ramanathan, Moduli for principal bundles over algebraic curves: I and II, Proc. Indian Acad. Sci. (Math. Sci.), 106 (1996), 301-328, 421-449.

[16] A.N. Tyurin, The classification of vector bundles over an algebraic curve of arbitrary genus, Izv. Akad. Nauk SSSR 29 (1965), 657-688.

[17] H. Umemura, On a theorem of Ramanan, Nagoya Math. J. 69 (1978), 131-138. 
School of Mathematics

Tata Institute of Fundamental Research

HOMI BHABHA ROAD

MUMBAI 400005

INDIA

E-mail address: indranil@math.tifr.res.in

Instituto de Ciencias Matemáticas (CSIC-UAM-UC3M-UCM)

Nicolas CABRERA 13

Campus Cantoblanco UAM

28049 MADRID AND

Facultad de Ciencias Matemáticas

Universidad Complutense De MAdRID

28040 MADRID

SPAIN

E-mail address: tomas.gomez@icmat.es

Received OCtober 28, 2009 